\title{
Unitarity of Time-Like Noncommutative Gauge Theories: The Violation of Ward Identities in Time-Ordered Perturbation Theory ${ }^{1}$
}

\author{
Thorsten Ohl Reinhold Rückl Jörg Zeiner \\ Institut für Theoretische Physik und Astrophysik, Universität Würzburg, \\ Am Hubland, 97074 Würzburg, Germany
}

\begin{abstract}
We study Ward identities for simple processes with external gauge bosons in the time-ordered perturbation theory approach to time-like noncommutative gauge theories. We demonstrate that these Ward identities cannot be satisfied when all orders in the noncommutativity parameters $\theta_{i 0}$ are taken into account. We conclude that in time-ordered perturbation theory one cannot solve the unitarity problem of timelike noncommutative quantum field theories.
\end{abstract}

Key words: noncommutative field theory, gauge theory, unitarity, Ward identities

\section{Introduction}

Noncommutative Quantum Field Theory (NCQFT) has recently received renewed attention (see [1] for a review). This interest is triggered by the connection of NCQFT to string theory, by its nature as a non-local generalization of Quantum Field Theory (QFT) and by the possibility of experimental tests, provided the scale of the noncommutativity is sufficiently small.

NCQFT starts from the assumption that the familiar continuous Minkowski space-time with coordinates $x_{\mu}$ is the long-distance limit of a space-time ge-

Email addresses: ohl@physik.uni-wuerzburg.de (Thorsten Ohl), rueckl@physik. uni-wuerzburg. de (Reinhold Rückl), zeiner@physik . uni-wuerzburg. de (Jörg Zeiner).

1 WUE-ITP-2003-005 [hep-th/0309021] (September 2003) 
ometry with noncommuting coordinates $\hat{x}_{\mu}$, satisfying commutation relations

$$
\left[\hat{x}_{\mu}, \hat{x}_{\nu}\right]=\frac{\mathrm{i} \theta_{\mu \nu}}{\Lambda^{2}}
$$

In general, the antisymmetric matrix $\theta_{\mu \nu}$ may depend on $\hat{x}_{\mu}$. In this note, like in most previous studies, $\theta_{\mu \nu}$ is assumed to be constant. The noncommuting coordinates can be realized on an ordinary commuting space-time by the associative Moyal $*$-product

$$
(f * g)(x)=\lim _{\xi, \eta \rightarrow 0}\left[\mathrm{e}^{\mathrm{i} \partial_{\xi} \wedge \partial_{\eta}} f(x+\xi) g(x+\eta)\right]
$$

which in momentum space induces phase factors $\mathrm{e}^{\mathrm{i} p \wedge q}$ with the antisymmetric product

$$
p \wedge q=\frac{1}{2 \Lambda^{2}} p^{\mu} \theta_{\mu \nu} q^{\nu} .
$$

In order to construct a NCQFT from a given QFT one can make use of a correspondence principle according to which all ordinary products of fields in the Lagrangian are replaced by Moyal *-products (see [1] and references cited therein). As a result, all interaction vertices acquire momentum-dependent phase factors.

The Moyal *-product (2) involves derivatives of all orders and thus makes the theory non-local. Great care has therefore to be taken that the physical interpretation of the theory is not spoiled by this non-locality. While it appears that the non-locality can be controlled for space-like noncommutativity, i. e. $\theta_{i j} \neq 0$ [2], there are serious problems with unitarity in the case of time-like noncommutativity, i. e. $\theta_{i 0} \neq 0$, when all orders in $\theta_{i 0}$ are taken into account. The perturbative $S$-matrix is no longer unitary, since the cutting rules are violated, as shown by an explicit calculation in the usual covariant perturbation theory [3].

In [4], Time-Ordered Perturbation Theory (TOPT) for NCQFT was introduced as an attempt to solve the unitarity problem [3] by constructing a manifestly unitary time evolution operator in non-covariant perturbation theory. In [5], it has then been demonstrated that TOPT indeed solves the unitarity problem for scalar fields in time-like NCQFT. Applications of TOPT to processes without external gauge bosons in Noncommutative Quantum Electrodynamics (NCQED) have been discussed in [6]. In section 2 of the present article, we briefly review TOPT in order to establish our notation and complete the Feynman rules given in $[4,6]$.

However, the formal unitarity of the time evolution operator does not suffice in covariantly quantized gauge theories, where unphysical degrees of freedom contribute negative norm states. The unphysical degrees of freedom must be proven to decouple from the $S$-matrix to obtain unitarity in the physical sub- 
space. In sections 3 and 4 we will show that the tree-level Ward Identities (WIs)

$$
\frac{\partial}{\partial x^{\mu}}\left\langle 0\left|\mathrm{~T} A^{\mu}(x) \Phi_{1}\left(x_{1}\right) \Phi_{2}\left(x_{2}\right) \cdots \Phi_{n}\left(x_{n}\right)\right| 0\right\rangle_{\text {amputated, on-shell }}=0
$$

with external photons cannot be satisfied in TOPT for time-like NCQED. The Green function (4) is amputated for all fields $\Phi_{i}$. The amputated gauge field $A_{\mu}$ corresponds to a current insertion.

A practical consequence of the violation of the WIs (4) is that differential cross sections calculated with covariant polarization sums - including sums over ghosts - are no longer positive definite. Indeed, we were alerted to the problem under investigation by this phenomenon in the calculation of Compton scattering. Furthermore, the violation of tree level WIs with external gauge bosons will obviously invalidate the cutting rules for loops involving gauge bosons.

Thus TOPT does not solve the unitarity problem in NCQED for processes with external photons. Our proof of this statement carries over to any timelike Noncommutative Gauge Theory (NCGT).

\section{Time-Ordered Perturbation Theory}

Upon integration over space-time, the *-product (2) is cyclically symmetric and reduces to the ordinary product for one pair of factors

$$
\begin{aligned}
\int \mathrm{d}^{4} x\left(f_{1} * f_{2} * \cdots * f_{n}\right)(x) & =\int \mathrm{d}^{4} x\left(f_{2} * \cdots * f_{n} * f_{1}\right)(x) \\
\int \mathrm{d}^{4} x\left(f_{1} * \cdots * f_{n}\right)(x) & =\int \mathrm{d}^{4} x\left(f_{1} * \cdots * f_{i}\right)(x)\left(f_{i+1} * \cdots * f_{n}\right)(x)
\end{aligned}
$$

This can be seen by partial integration, using the antisymmetry of (3). It follows from (5b) that the propagators remain unchanged when ordinary products are replaced by $*$-products. In addition, (5a) shows that the additional phases are invariant under cyclic permutations.

In [4] it has been proposed to use TOPT to construct formally unitary quan-

tum field theories with time-like noncommutativity. In TOPT, each propaga- 
tor is split into a positive energy and negative energy piece:

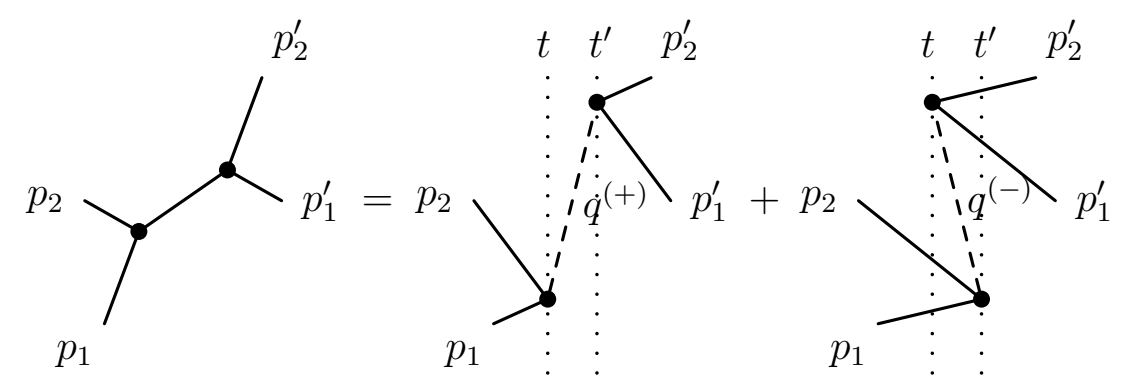

Here all four-momenta are taken on-shell, in particular

$$
q^{( \pm)}=\left( \pm \sqrt{\vec{q}^{2}+m^{2}}, \vec{q}\right)
$$

Since the three-momentaare conserved at a vertex, $\vec{q}=\vec{p}_{1}+\vec{p}_{2}=\vec{p}_{1}^{\prime}+\vec{p}_{2}^{\prime}$, energy cannot be conserved.

After Fourier transformation (F.T.), the Moyal phase $\varphi$ of a $n$-point vertex

$$
\left(\Phi_{1} * \Phi_{2} * \cdots * \Phi_{n}\right)(x) \stackrel{\mathrm{F} . \mathrm{T}}{\longrightarrow} \mathrm{e}^{-\mathrm{i} \varphi\left(p_{1}, p_{2}, \ldots, p_{n}\right)} \Phi_{1}\left(p_{1}\right) \Phi_{2}\left(p_{2}\right) \cdots \Phi_{n}\left(p_{n}\right)
$$

is given by

$$
\varphi\left(p_{1}, p_{2}, \ldots, p_{n}\right)=\sum_{i<j} p_{i} \wedge p_{j}
$$

using the notation (3). The phase $\varphi$ defined in (9) is not cyclically symmetric

$$
\varphi\left(p_{1}, p_{2}, \ldots, p_{n}\right) \neq \varphi\left(p_{2}, \ldots, p_{n}, p_{1}\right) .
$$

Only in the case of four-momentum conservation, the contributions from either the first or the last momentum cancel

$$
\left.\varphi\left(p_{1}, p_{2}, \ldots, p_{n}\right)\right|_{p_{1}+p_{2}+\cdots+p_{n}=0}=\varphi\left(p_{1}, p_{2}, \ldots, p_{n-1}\right)=\varphi\left(p_{2}, \ldots, p_{n}\right)
$$

and cyclical symmetry is recovered. Therefore, (10) introduces an ordering ambiguity in (8) that must be taken into account.

Using the notation $q_{0}^{( \pm)}= \pm \sqrt{\vec{q}^{2}+m^{2}}$, the two scalar propagators in TOPT can be expressed as [4]

$$
-------\quad=\frac{\mathrm{i}}{2 q_{0}^{(\lambda)}} \frac{1}{q_{0}-q_{0}^{(\lambda)}+\lambda \mathrm{i} \epsilon} .
$$

Since the phase (9) depends on all four-momenta at the vertex, it is generally impossible to remove the dependence of $\varphi$ on the two on-shell momenta $q^{( \pm)}$ with positive and negative energy. Consequently, the two contributions to the scattering process in (6) have different Moyal phases $\phi\left(q^{( \pm)}\right)$, where the 
dependence on the external four-momenta is suppressed for brevity. After adding up these two contributions, the same poles as in covariant perturbation theory appear

$$
\sum_{\lambda= \pm} \mathrm{e}^{\mathrm{i} \phi\left(q^{(\lambda)}\right)} \frac{1}{2 q_{0}^{(\lambda)}} \frac{1}{q_{0}-q_{0}^{(\lambda)}+\lambda \mathrm{i} \epsilon}=\frac{R\left(q^{(+)}, q^{(-)}\right)}{q_{0}^{2}-\vec{q}^{2}-m^{2}+\mathrm{i} \varepsilon}=\frac{R\left(q^{(+)}, q^{(-)}\right)}{q^{2}-m^{2}+\mathrm{i} \varepsilon}
$$

however the residue is not simply unity, but a linear combination of the phase factors $\mathrm{e}^{\mathrm{i} \phi\left(q^{( \pm)}\right)}[4]$ :

$$
R\left(q^{(+)}, q^{(-)}\right)=\frac{1}{2} \sum_{\lambda= \pm} \mathrm{e}^{\mathrm{i} \phi\left(q^{(\lambda)}\right)}\left(1+\frac{q_{0}}{q_{0}^{(\lambda)}}\right) .
$$

At this point we want to stress that the structure of (13) allows the following approach to WIs: in a first step, we can ignore the phases from TOPT and draw conclusions from the required cancellation of poles alone. In the second step, we can then use the results from the first requirement and derive relations among the phases.

The authors of [4] have not given a prescription for propagators with momenta in the numerator. This will be done here. The propagator for a spin- $1 / 2$ field, is composed of the positive and negative energy contributions

$$
\begin{aligned}
S^{(+)}(x) & =\int \frac{\mathrm{d}^{3} \vec{q}}{(2 \pi)^{3} 2 q_{0}^{(+)}} \sum_{s= \pm} u_{s}(q) \bar{u}_{s}(q) e^{-\mathrm{i} q^{(+)} x} \\
& =\int \frac{\mathrm{d}^{3} \vec{q}}{(2 \pi)^{3} 2 q_{0}^{(+)}}\left(\phi^{(+)}+m\right) e^{-\mathrm{i} q^{(+)} x} \\
S^{(-)}(x) & =\int \frac{\mathrm{d}^{3} \vec{q}}{(2 \pi)^{3} 2 q_{0}^{(+)}} \sum_{s= \pm} v_{s}(q) \bar{v}_{s}(q) e^{\mathrm{i} q^{(+)} x}=\int \frac{\mathrm{d}^{3} \vec{q}}{(2 \pi)^{3} 2 q_{0}^{(+)}}\left(\phi^{(+)}-m\right) e^{\mathrm{i} q^{(+)} x} \\
& =\int \frac{\mathrm{d}^{3} \vec{q}}{(2 \pi)^{3} 2 q_{0}^{(-)}}\left(\phi^{(-)}+m\right) e^{-\mathrm{i} q^{(-)} x}
\end{aligned}
$$

where we have substituted $\vec{q} \rightarrow-\vec{q}$ in the last step of (15b). Hence the spin- $1 / 2$ propagators in momentum space in TOPT are given by

$$
\overrightarrow{q^{(\lambda)}}=\frac{\mathrm{i}}{2 q_{0}^{(\lambda)}} \frac{q^{(\lambda)}+m}{q_{0}-q_{0}^{(\lambda)}+\lambda \mathrm{i} \epsilon} .
$$

Just as for scalar particles, the poles are the same in TOPT as in covariant perturbation theory, but the residue is modified and a regular term is added:

$$
\sum_{\lambda= \pm} \mathrm{e}^{\mathrm{i} \phi\left(q^{(\lambda)}\right)} \frac{1}{2 q_{0}^{(\lambda)}} \frac{\phi^{(\lambda)}+m}{q_{0}-q_{0}^{(\lambda)}+\lambda \mathrm{i} \epsilon}=\frac{1}{q^{2}-m^{2}+\mathrm{i} \varepsilon} \times
$$




$$
\begin{array}{r}
\left(R_{+}\left(q^{(+)}, q^{(-)}\right)(\not q+m)+R_{-}\left(q^{(+)}, q^{(-)}\right)\left(\gamma^{0} q_{0}^{(+)}-\frac{q_{0}}{q_{0}^{(+)}}(\vec{\gamma} \vec{q}-m)\right)\right) \\
=\frac{R\left(q^{(+)}, q^{(-)}\right)}{\not 1-m+\mathrm{i} \varepsilon}-\gamma^{0} \frac{R_{-}\left(q^{(+)}, q^{(-)}\right)}{q_{0}^{(+)}}
\end{array}
$$

with $R$ from (14) and

$$
R_{ \pm}\left(q^{(+)}, q^{(-)}\right)=\frac{1}{2}\left(\mathrm{e}^{\mathrm{i} \phi\left(q^{(+)}\right)} \pm \mathrm{e}^{\mathrm{i} \phi\left(q^{(-)}\right)}\right)
$$

The gauge boson propagator in TOTP can be derived analogously. For our purpose it suffices to consider only Feynman gauge $(\xi=1)$, in which case there are no momenta in the numerator and the propagator is given by

$$
\sim \sim_{q^{(\lambda)}}^{\sim} \sim=\frac{-\mathrm{i} g_{\mu \nu}}{2 q_{0}^{(\lambda)}} \frac{1}{q_{0}-q_{0}^{(\lambda)}+\lambda \mathrm{i} \epsilon}
$$

\section{$2.1 N C Q E D$}

The arguments that will be put forward in section 3 are valid for arbitrary $\mathrm{U}(N)$ NCGTs. For $N=1$ all nonzero charges have to be the same, up to a sign. NCGTs for $\mathrm{SU}(N)$ and $\mathrm{U}(1)$ with different nonzero charges can be constructed in the enveloping algebra [7]. The investigation of the interplay of gauge invariance, temporal non-locality and unitarity in the formulation [7] requires the study of the corresponding Seiberg-Witten maps to all orders in $\theta_{\mu \nu}$ and will be the subject of future research. For simplicity, we confine ourselves to a U(1)-theory with one massive spin-1/2 matter field (NCQED). It will be obvious how to generalize the results.

The Lagrangian for NCQED,

$$
\mathcal{L}=-\frac{1}{4} F_{\mu \nu} * F_{\mu \nu}+\bar{\psi} *(\mathrm{i} \not D-m) * \psi,
$$

with

$$
\begin{aligned}
D_{\mu} & =\partial_{\mu}-\mathrm{i} e A_{\mu} \\
F_{\mu \nu} & =\frac{\mathrm{i}}{e}\left[D_{\mu}, D_{\nu}\right]=\partial_{\mu} A_{\nu}-\partial_{\nu} A_{\mu}-\mathrm{i} e\left[A_{\mu}, A_{\nu}\right]
\end{aligned}
$$

is invariant under the gauge transformations

$$
\begin{aligned}
& \delta_{\eta} \psi=\mathrm{i} e \eta * \psi \\
& \delta_{\eta} \bar{\psi}=-\mathrm{i} e \bar{\psi} * \eta
\end{aligned}
$$




$$
\delta_{\eta} A_{\mu}=\left[D_{\mu}^{*}, \eta\right]
$$

where the $*$-commutator is defined by

$$
[f * g]=f * g-g * f .
$$

The gauge invariance can most easily be seen from the covariant transformation rules

$$
\begin{aligned}
\delta_{\eta} D_{\mu} & =-\mathrm{i} e \delta_{\eta} A_{\mu}=\mathrm{i} e\left[\eta * D_{\mu}\right] \\
\delta_{\eta}\left(D_{\mu} * \psi\right) & =\mathrm{i} e \eta * D_{\mu} * \psi \\
\delta_{\eta} F_{\mu \nu} & =\mathrm{i} e\left[\eta * F_{\mu \nu}\right] .
\end{aligned}
$$

The cubic and quartic interactions in the Lagrangian (20) are given by

$$
\begin{aligned}
& \mathcal{L}_{3}=e \bar{\psi} * A * \psi+e \mathrm{i} \partial_{\mu} A_{\nu} *\left[A^{\mu}, A^{\nu}\right] \\
& \mathcal{L}_{4}=\frac{e^{2}}{4}\left[A_{\mu} * A_{\nu}\right] *\left[A^{\mu}, A^{\nu}\right] .
\end{aligned}
$$

Taking into account the ambiguity (10) in assigning the Moyal phases $\varphi$ in TOPT, one has for the $e^{+} e^{-} \gamma$-vertex:

$$
\begin{array}{r}
\bar{\psi} * A * \psi \rightarrow\left(c_{1} \bar{\psi}_{\alpha} * A_{\mu} * \psi_{\beta}+c_{2} A_{\mu} * \psi_{\beta} * \bar{\psi}_{\alpha}+c_{3} \psi_{\beta} * \bar{\psi}_{\alpha} * A_{\mu}\right) \gamma_{\alpha \beta}^{\mu} \\
\stackrel{\text { F.T. }}{\longrightarrow} \sum_{i=1}^{3} c_{i} \mathrm{e}^{-\mathrm{i} \varphi_{i}(\bar{p}, k, p)} \bar{\psi}(\bar{p}) A(k) \psi(p)
\end{array}
$$

with arbitrary coefficients $c_{i}$ obeying $c_{1}+c_{2}+c_{3}=1$. Here we have introduced the notation $\varphi_{l}\left(k_{1}, k_{2}, k_{3}\right)=\varphi\left(k_{l}, k_{m}, k_{n}\right)$ for cyclical permutations $\{l, m, n\}$ of $\{1,2,3\}$. With $p$ and $k$ incoming and $p^{\prime}$ outgoing, the corresponding vertex factor is given by

$$
\left.p^{\prime}-p=k\right\} p^{\prime}=\mathrm{i} e \gamma_{\mu} \sum_{i=1}^{3} c_{i} \mathrm{e}^{-\mathrm{i} \varphi_{i}\left(-p^{\prime}, k, p\right)}
$$

While the phase factors in (26) and (27) are fixed by TOPT, the choice of the coefficients $c_{i}$ is ambiguous. In the case of the $3 \gamma$-vertex

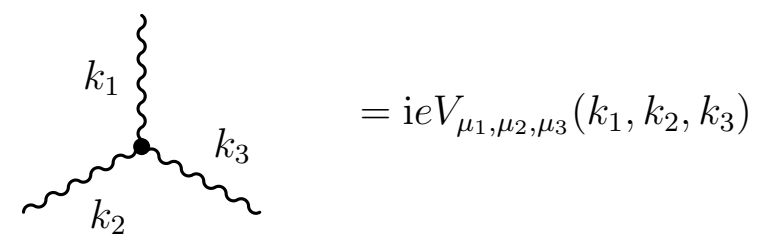


the corresponding ambiguity in the Moyal phases leads to

$$
\begin{aligned}
c_{1}^{\prime} \mathrm{i} \partial_{\mu} A_{\nu} & * A^{\mu} * A^{\nu}+c_{2}^{\prime} A^{\mu} * A^{\nu} * \mathrm{i} \partial_{\mu} A_{\nu}+c_{3}^{\prime} A^{\nu} * \mathrm{i} \partial_{\mu} A_{\nu} * A^{\mu} \\
& -c_{1}^{\prime} \mathrm{i} \partial_{\mu} A_{\nu} * A^{\nu} * A^{\mu}-c_{2}^{\prime} A^{\nu} * A^{\mu} * \mathrm{i} \partial_{\mu} A_{\nu}-c_{3}^{\prime} A^{\mu} * \mathrm{i} \partial_{\mu} A_{\nu} * A^{\nu}
\end{aligned}
$$

with $c_{1}^{\prime}+c_{2}^{\prime}+c_{3}^{\prime}=1$. In general, the coefficients $c_{i}^{\prime}$ and $c_{i}$ are different.

When Fourier transforming (29), one faces another ambiguity associated with the derivative couplings. In covariant perturbation theory, derivatives can be shifted by partial integration from one field to the other fields at the same vertex, with energy-momentum conservation insuring that the final result is independent of this choice. In TOPT however, one will get different results from a derivative acting on one field and a derivative acting on the product of the other fields. While in a local QFT the physical results can be shown to be equivalent by using the equations of motion [8], this is not the case in a noncommutative QFT with an infinite number of time derivatives. In section 3 we will use the WIs to derive an unambiguous prescription for the choice of the momenta corresponding to the derivatives after Fourier transformation. These momenta are denoted by $\bar{k}_{i}$ for now.

With this convention the interaction (29) yields the vertex factor

$$
\begin{aligned}
& \mathrm{i} V_{\mu_{1}, \mu_{2}, \mu_{3}}\left(k_{1}, k_{2}, k_{3}\right)= \\
& \sum_{i=1}^{3} c_{i}^{\prime}\left(\bar{k}_{1}^{\mu_{2}} g^{\mu_{1} \mu_{3}}-\bar{k}_{1}^{\mu_{3}} g^{\mu_{1} \mu_{2}}\right)\left(\mathrm{e}^{-\mathrm{i} \varphi_{i}\left(k_{1}, k_{2}, k_{3}\right)}-\mathrm{e}^{-\mathrm{i} \varphi_{i}\left(k_{1}, k_{3}, k_{2}\right)}\right) \\
&+\operatorname{cyclic}\{1,2,3\} .
\end{aligned}
$$

where $k_{1,2,3}$ appearing in the Moyal phases are the on-shell momenta of TOPT. Defining

$$
\delta k=k_{1}+k_{2}+k_{3}
$$

the expression (30) can be written in the form

$$
\mathrm{i} V_{\mu_{1}, \mu_{2}, \mu_{3}}\left(k_{1}, k_{2}, k_{3}\right)=\left(\bar{k}_{1}^{\mu_{2}} g^{\mu_{1} \mu_{3}}-\bar{k}_{1}^{\mu_{3}} g^{\mu_{1} \mu_{2}}\right) \mathcal{C}_{23}\left(k_{1}, k_{2}, k_{3}\right)+\operatorname{cyclic}\{1,2,3\}
$$

with

$$
\mathcal{C}_{23}\left(k_{1}, k_{2}, k_{3}\right)=2 \sin \left(k_{2} \wedge k_{3}\right) C_{1}\left(k_{1}, k_{2}, k_{3}\right)+2 C_{0}\left(k_{1}, k_{2}, k_{3}\right)
$$

and

$$
\begin{aligned}
& C_{1}\left(k_{1}, k_{2}, k_{3}\right)=c_{1}^{\prime} \mathrm{e}^{-\mathrm{i} k_{1} \wedge \delta k}+c_{2}^{\prime} \mathrm{e}^{\mathrm{i} k_{1} \wedge \delta k}+c_{3}^{\prime} \cos \left(\delta k \wedge\left(k_{2}-k_{3}\right)\right) \\
& C_{0}\left(k_{1}, k_{2}, k_{3}\right)=c_{3}^{\prime} \cos \left(k_{2} \wedge k_{3}\right) \sin \left(\delta k \wedge\left(k_{2}-k_{3}\right)\right)
\end{aligned}
$$

Note that

$$
\left.\mathcal{C}_{23}\left(k_{1}, k_{2}, k_{3}\right)\right|_{\delta k=0}=2 \sin \left(k_{2} \wedge k_{3}\right)
$$


as in NCQED for $\theta_{i j} \neq 0[9]$.

\section{Ward Identities for Compton Scattering}

After adding the gauge-fixing term

$$
\mathcal{L}_{\text {g.f. }}=\delta_{\text {BRST }}\left(\bar{c} *\left(\frac{\xi}{2} B+\partial_{\mu} A^{\mu}\right)\right)
$$

with Faddeev-Popov ghosts $c$, antighosts $\bar{c}$ and a Nakanishi-Lautrup field $B$, to the Lagrangian (20), the sum $\mathcal{L}+\mathcal{L}_{\text {g.f. }}$ is invariant under the BRST transformations [10]

$$
\begin{aligned}
\delta_{\mathrm{BRST}} \psi & =\mathrm{i} e c * \psi \\
\delta_{\mathrm{BRST}} \bar{\psi} & =-\mathrm{i} e \bar{\psi} * c \\
\delta_{\mathrm{BRST}} A_{\mu} & =\left[D_{\mu} * c\right] \\
\delta_{\mathrm{BRST}} c & =\mathrm{i}[c * c] \\
\delta_{\mathrm{BRST}} \bar{c} & =B \\
\delta_{\mathrm{BRST}} B & =0 .
\end{aligned}
$$

The invariance of the action under (36) engenders relations among Green functions of the theory, known as Slavnov-Taylor identities (STIs). The prototype STI is derived from

$$
\left\langle 0\left|\operatorname{T} \delta_{\operatorname{BRST}}\left(\bar{c}(x) \Phi_{1}\left(x_{1}\right) \Phi_{2}\left(x_{2}\right) \cdots \Phi_{n}\left(x_{n}\right)\right)\right| 0\right\rangle=0
$$

using the equation of motion for $B=-\partial_{\mu} A^{\mu} / \xi$ :

$$
\begin{aligned}
& \frac{\partial}{\partial x^{\mu}}\left\langle 0\left|\mathrm{~T} A^{\mu}(x) \Phi_{1}\left(x_{1}\right) \Phi_{2}\left(x_{2}\right) \cdots \Phi_{n}\left(x_{n}\right)\right| 0\right\rangle= \\
& \xi \sum_{i}( \pm)\left\langle 0\left|\mathrm{~T} \bar{c}(x) \Phi_{1}\left(x_{1}\right) \cdots \delta_{\mathrm{BRST}} \Phi_{i}\left(x_{i}\right) \cdots \Phi_{n}\left(x_{n}\right)\right| 0\right\rangle
\end{aligned}
$$

where the sign of each summand is fixed by the anticommuting nature of the BRST transformation.

In (36) the BRST transforms of the physical degrees of freedom $\psi, \bar{\psi}$ and $A_{\mu}$ (with $\partial_{\mu} A^{\mu}=0$ ) are bilinear in these fields and the ghost $c$. Therefore, the contributions of these transforms to the STIs are cancelled when matrix elements of physical fields are amputated on-shell. As a consequence, the STIs (38) reduce on-shell to the WIs

$$
\frac{\partial}{\partial x^{\mu}}\left\langle 0\left|\mathrm{~T} A^{\mu}(x) \Phi_{1}\left(x_{1}\right) \Phi_{2}\left(x_{2}\right) \cdots \Phi_{n}\left(x_{n}\right)\right| 0\right\rangle_{\text {amputated, on-shell }}=0
$$


if all fields $\Phi_{j}$ are either matter fields or gauge fields with physical polarizations.

When any one of the WIs (4) is violated, the BRST charge $Q_{\mathrm{BRST}}$ generating (36) is not conserved and it is impossible to construct a positive norm Hilbert space for the physical asymptotic states from the cohomology of the BRST operator using the condition $Q_{\mathrm{BRST}} \mid$ phys $\rangle=0$. Therefore, one cannot not make physical sense of a gauge theory, unless the WIs (4) are satisfied.

The simplest example for the violation of WIs in TOPT is provided by Compton scattering $e^{-} \gamma \rightarrow e^{-} \gamma$ or any of its crossed variants. The three contributing Feynman diagrams

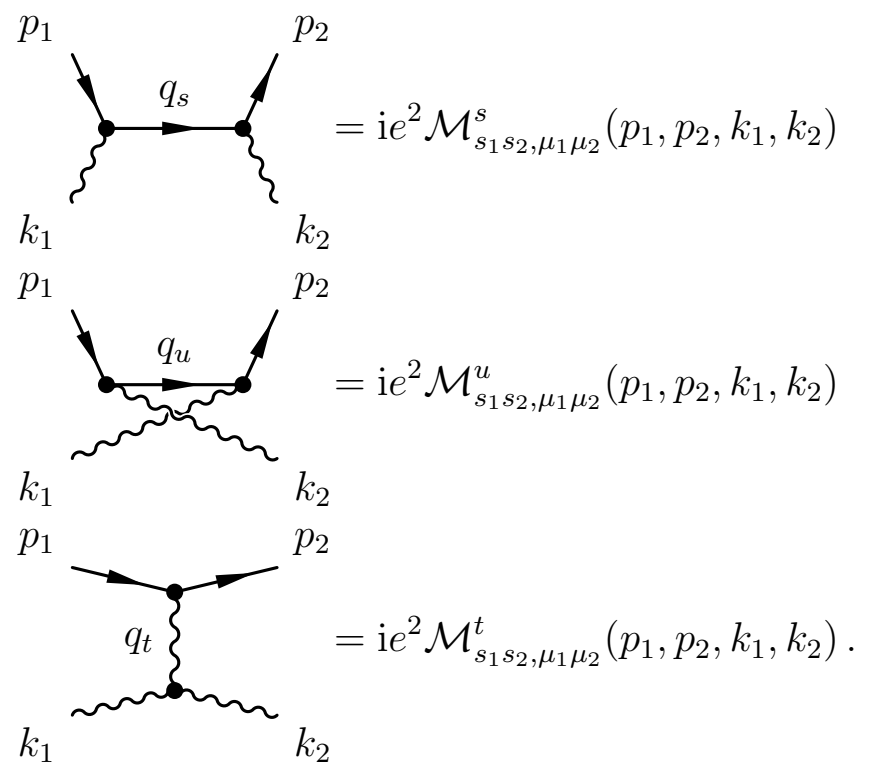

yield

$$
\begin{aligned}
k_{1}^{\mu_{1}}\left\langle 0\left|\mathrm{~T} A_{\mu_{1}}\left(k_{1}\right)\left(\epsilon_{(\kappa)}^{\mu_{2}}\left(k_{2}\right) A_{\mu_{2}}\left(k_{2}\right)\right) \psi_{1}\left(p_{1}\right) \bar{\psi}_{2}\left(p_{2}\right)\right|\right. & 0\rangle_{\text {amputated, on-shell }} \\
& =\mathcal{W}_{(\kappa)}^{s}+\mathcal{W}_{(\kappa)}^{u}+\mathcal{W}_{(\kappa)}^{t}
\end{aligned}
$$

for photon polarizations $\kappa= \pm$. Starting with the $s$-channel and defining

$$
\begin{aligned}
q_{s} & =p_{1}+k_{1}=p_{2}+k_{2} \\
q_{u} & =p_{1}-k_{2}=p_{2}-k_{1} \\
q_{t} & =p_{2}-p_{1}=k_{1}-k_{2},
\end{aligned}
$$

we find

$$
\mathcal{W}_{(\kappa)}^{s}=k_{1}^{\mu_{1}} \epsilon_{(\kappa)}^{\mu_{2}}\left(k_{2}\right) \mathcal{M}_{s_{1} s_{2}, \mu_{1} \mu_{2}}^{s}\left(p_{1}, p_{2}, k_{1}, k_{2}\right)=\mathcal{W}_{(\kappa)}^{s, 1}+\mathcal{W}_{(\kappa)}^{s, 0}
$$


with

$$
\begin{aligned}
\mathcal{W}_{(\kappa)}^{s, 1} & =\sum_{i, j=1}^{3} c_{i} c_{j} R^{s, i j}\left(q_{s}^{(+)}, q_{s}^{(-)}\right) \bar{u}_{s_{2}}\left(p_{2}\right) \notin_{(\kappa)}\left(k_{2}\right) u_{s_{1}}\left(p_{1}\right) \\
\mathcal{W}_{(\kappa)}^{s, 0} & =-\sum_{i, j=1}^{3} c_{i} c_{j} R_{-}^{s, i j}\left(q_{s}^{(+)}, q_{s}^{(-)}\right) \frac{1}{q_{s, 0}^{(+)}} \bar{u}_{s_{2}}\left(p_{2}\right) \notin_{(\kappa)}\left(k_{2}\right) \gamma_{0} \not k_{1} u_{s_{1}}\left(p_{1}\right) .
\end{aligned}
$$

and, using (14) and (18),

$$
\begin{aligned}
& R^{s, i j}\left(q_{s}^{(+)}, q_{s}^{(-)}\right)=\sum_{\lambda= \pm} \mathrm{e}^{-\mathrm{i} \varphi_{i}\left(-p_{2},-k_{2}, q_{s}^{(\lambda)}\right)} \mathrm{e}^{-\mathrm{i} \varphi_{j}\left(-q_{s}^{(\lambda)}, k_{1}, p_{1}\right)} \frac{1}{2}\left(1+\frac{q_{s, 0}}{q_{s}^{(\lambda)}}\right) \\
& R_{-}^{s, i j}\left(q_{s}^{(+)}, q_{s}^{(-)}\right)=\sum_{\lambda= \pm} \mathrm{e}^{-\mathrm{i} \varphi_{i}\left(-p_{2},-k_{2}, q_{s}^{(\lambda)}\right)} \mathrm{e}^{-\mathrm{i} \varphi_{j}\left(-q_{s}^{(\lambda)}, k_{1}, p_{1}\right)} \frac{\lambda}{2}
\end{aligned}
$$

Here, the term $\mathcal{W}_{(\kappa)}^{s, 0}$ remains after using the equation of motion for cancelling the electron propagator

$$
\frac{1}{\not q_{s}-m+\mathrm{i} \varepsilon} \not k_{1} u_{s_{1}}\left(p_{1}\right)=\frac{1}{\not q_{s}-m+\mathrm{i} \varepsilon}\left(\not p_{1}+\not k_{1}-m\right) u_{s_{1}}\left(p_{1}\right)=u_{s_{1}}\left(p_{1}\right) \text {. }
$$

Similarly for the $u$-channel, one gets

$$
\mathcal{W}_{(\kappa)}^{u}=k_{1}^{\mu_{1}} \epsilon_{(\kappa)}^{\mu_{2}}\left(k_{2}\right) \mathcal{M}_{s_{1} s_{2}, \mu_{1} \mu_{2}}^{u}\left(p_{1}, p_{2}, k_{1}, k_{2}\right)=\mathcal{W}_{(\kappa)}^{u, 1}+\mathcal{W}_{(\kappa)}^{u, 0}
$$

with

$$
\begin{aligned}
& \mathcal{W}_{(\kappa)}^{u, 1}=-\sum_{i, j=1}^{3} c_{i} c_{j} R^{u, i j}\left(q_{u}^{(+)}, q_{u}^{(-)}\right) \bar{u}_{s_{2}}\left(p_{2}\right) \notin(\kappa) \\
& \mathcal{W}_{(\kappa)}^{u, 0}=-\sum_{i, j=1}^{3} c_{i} c_{j} R_{-}^{u, i j}\left(q_{u}^{(+)}, q_{s_{1}}\left(p_{1}\right)\right.
\end{aligned}
$$

and

$$
\begin{aligned}
& R^{u, i j}\left(q_{u}^{(+)}, q_{u}^{(-)}\right)=\sum_{\lambda= \pm} \mathrm{e}^{-\mathrm{i} \varphi_{i}\left(-p_{2}, k_{1}, q_{u}^{(\lambda)}\right)} \mathrm{e}^{-\mathrm{i} \varphi_{j}\left(-q_{u}^{(\lambda)},-k_{2}, p_{1}\right)} \frac{1}{2}\left(1+\frac{q_{u, 0}}{q_{u, 0}^{(\lambda)}}\right) \\
& R_{-}^{u, i j}\left(q_{u}^{(+)}, q_{u}^{(-)}\right)=\sum_{\lambda= \pm} \mathrm{e}^{-\mathrm{i} \varphi_{i}\left(-p_{2}, k_{1}, q_{u}^{(\lambda)}\right)} \mathrm{e}^{-\mathrm{i} \varphi_{j}\left(-q_{u}^{(\lambda)},-k_{2}, p_{1}\right)} \frac{\lambda}{2} .
\end{aligned}
$$

Finally, the $t$-channel contribution is given by

$$
\begin{aligned}
\mathcal{W}_{(\kappa)}^{t}=k_{1}^{\mu_{1}} \epsilon_{(\kappa)}^{\mu_{2}}\left(k_{2}\right) & \mathcal{M}_{s_{1} s_{2}, \mu_{1} \mu_{2}}^{t}\left(p_{1}, p_{2}, k_{1}, k_{2}\right) \\
= & \sum_{i=1}^{3} c_{i} \sum_{\lambda= \pm} \mathrm{e}^{-\mathrm{i} \varphi_{i}\left(-p_{2}, q_{t}^{(\lambda)}, p_{1}\right)} \frac{1}{2}\left(1+\frac{q_{u, 0}}{q_{u, 0}^{(\lambda)}}\right) \times \\
& \bar{u}_{s_{2}}\left(p_{2}\right) \gamma^{\mu_{3}} u_{s_{1}}\left(p_{1}\right) \frac{1}{q_{t}^{2}} V_{\mu_{1} \mu_{2} \mu_{3}}\left(k_{1},-k_{2},-q_{t}^{(\lambda)}\right) k_{1}^{\mu_{1}} \epsilon_{(\kappa)}^{\mu_{2}}\left(k_{2}\right)
\end{aligned}
$$


where the additional phases are absorbed in the $3 \gamma$ vertex factor $V$.

In the case of vanishing time-like noncommutativity, i. e. $\theta_{0 i}=0$, we can use (11) to remove all dependence of the Moyal phases on the internal momenta. Then the sums over $i, j$ and $\lambda$ become trivial, yielding

$$
\begin{aligned}
& \mathcal{W}_{(\kappa)}^{s}=\mathrm{e}^{-\mathrm{i} \varphi\left(-p_{2},-k_{2}, k_{1}, p_{1}\right)} \bar{u}_{s_{2}}\left(p_{2}\right) \notin(\kappa) \\
& \mathcal{W}_{(\kappa)}^{u}=-\mathrm{e}^{-\mathrm{i} \varphi\left(-p_{2}, k_{1},-k_{2}, p_{1}\right)} \bar{u}_{s_{2}}\left(p_{2}\right) \notin(\kappa) \\
& \mathcal{W}_{(\kappa)}^{t}=\mathrm{e}^{-\mathrm{i} \varphi\left(p_{1},-p_{2}\right)} \bar{u}_{s_{2}}\left(p_{2}\right) u_{s_{1}}\left(p_{1}\right) \\
& \gamma^{\mu_{3}} u_{s_{1}}\left(p_{1}\right) \frac{1}{q_{t}^{2}} V_{\mu_{1} \mu_{2} \mu_{3}}\left(k_{1},-k_{2},-q_{t}\right) k_{1}^{\mu_{1}} \epsilon_{(\kappa)}^{\mu_{2}}\left(k_{2}\right) .
\end{aligned}
$$

Furthermore, the $s$ - and $u$-channel contributions can be combined since the overall energy conservation makes the phases cyclically symmetric

$$
\mathcal{W}_{(\kappa)}^{s}+\mathcal{W}_{(\kappa)}^{u}=-2 \mathrm{i} \sin \left(k_{1} \wedge k_{2}\right) \mathrm{e}^{\mathrm{i} p_{1} \wedge p_{2}} \bar{u}_{s_{2}}\left(p_{2}\right) \notin_{(\kappa)}\left(k_{2}\right) u_{s_{1}}\left(p_{1}\right)
$$

Using

$k_{1}^{\mu_{1}} \epsilon_{(\kappa)}^{\mu_{2}}\left(k_{2}\right) V_{\mu_{1} \mu_{2} \mu_{3}}\left(k_{1},-k_{2},-q_{t}\right)=\mathrm{i}\left(q_{t}^{2}\left(_{(\kappa)}\left(k_{2}\right)-\left(q_{t} \epsilon_{(\kappa)}\left(k_{2}\right)\right) q_{t, \mu_{3}}\right) 2 \sin \left(k_{1} \wedge k_{2}\right)\right.$

one finally recovers $\mathcal{W}_{(\kappa)}^{s}+\mathcal{W}_{(\kappa)}^{t}+\mathcal{W}_{(\kappa)}^{u}=0[9]$

Returning to the general case, the presence of the $1 / q_{t}^{2}$ pole in (45), together with its absence in (42) and (44), shows that, for any cancellation between $\mathcal{W}_{(\kappa)}^{s}+\mathcal{W}_{(\kappa)}^{u}$ and $\mathcal{W}_{(\kappa)}^{t}$ to take place, we must have

$$
k_{1}^{\mu_{1}} \epsilon_{(\kappa)}^{\mu_{2}}\left(k_{2}\right) V_{\mu_{1} \mu_{2} \mu_{3}}\left(k_{1},-k_{2},-q_{t}^{(\lambda)}\right)=\alpha_{1} q_{t}^{2} \epsilon_{(\kappa), \mu_{3}}+\alpha_{2} q_{t, \mu_{3}},
$$

where the $\alpha_{2}$-term is allowed because it will not contribute due to current conservation, i. e. $\bar{u}\left(p_{2}\right) \phi_{t} u\left(p_{1}\right)=0$. In order to determine the consequences of (49), we can make the general ansatz

$$
\begin{aligned}
\tilde{V}_{\mu_{1} \mu_{2} \mu_{3}}\left(b_{1}, b_{2}, b_{3} \mid k_{1},\right. & \left.k_{2}, k_{3}\right)=\left(b_{1} \bar{k}_{1, \mu_{3}}-b_{2} \bar{k}_{2, \mu_{3}}\right) g_{\mu_{1} \mu_{2}} \\
& +\left(b_{2} \bar{k}_{2, \mu_{1}}-b_{3} \bar{k}_{3, \mu_{1}}\right) g_{\mu_{2} \mu_{3}}+\left(b_{3} \bar{k}_{3, \mu_{2}}-b_{1} \bar{k}_{1, \mu_{2}}\right) g_{\mu_{3} \mu_{1}}
\end{aligned}
$$

where the coefficients $b_{i}$ can contain momentum dependent phase factors. For the process at hand, we have $\bar{k}_{1}=k_{1}$ and $\bar{k}_{2}=k_{2}$ since they are external on-shell momenta $\left(k_{1}^{2}=k_{2}^{2}=0\right)$. Using $\epsilon_{(\kappa)}^{\mu}\left(k_{2}\right) k_{2, \mu}=0$, we obtain

$$
\begin{aligned}
& k_{1}^{\mu_{1}} \epsilon_{(\kappa)}^{\mu_{2}}\left(k_{2}\right) \tilde{V}_{\mu_{1} \mu_{2} \mu_{3}}\left(b_{1}, b_{2}, b_{3} \mid k_{1}, k_{2}, k_{3}\right)= \\
& \frac{b_{3}+b_{2}}{2} \bar{k}_{3}^{2} \epsilon_{(\kappa), \mu_{3}}\left(k_{2}\right)-b\left(\bar{k}_{3} \epsilon_{(\kappa)}\left(k_{2}\right)\right) \bar{k}_{3, \mu_{3}}+\left(\bar{k}_{3} \epsilon_{(\kappa)}\left(k_{2}\right)\right)\left(b_{3} k_{1}+b_{2} k_{2}+b \bar{k}_{3}\right)_{\mu_{3}} \\
& \quad-b_{2}\left(\delta \bar{k} \epsilon_{(\kappa)}\right) k_{2, \mu_{3}}+\left(\delta \bar{k}\left(b_{3} k_{2}-b_{2} \bar{k}_{3}\right)\right) \epsilon_{(\kappa), \mu_{3}}+\frac{b_{2}-b_{3}}{2}(\delta \bar{k})^{2} \epsilon_{(\kappa), \mu_{3}}, \quad
\end{aligned}
$$

where the term proportional to $b$ has been added and subtracted. The first term in the right hand side of (51) corresponds to the term proportional to $\alpha_{1}$ 
in (49) and cancels the pole. Like the term proportional to $\alpha_{2}$ in (49), the second term will not contribute to the WI. The remaining terms have to vanish. This gives us two conditions for the momenta in the derivative couplings

$$
\begin{gathered}
\delta \bar{k}=k_{1}+k_{2}+\bar{k}_{3}=0 \\
b_{3} k_{1}+b_{2} k_{2}+b \bar{k}_{3}=0,
\end{gathered}
$$

which can be satisfied simultaneously for $k_{i} \neq 0$, if and only if

$$
b_{3}=b_{2}=b .
$$

Therefore these momenta must satisfy energy-momentum conservation and cannot be the momenta in TOPT that do not conserve energy at the vertices.

At this point, we could proceed by attempting to solve the WI $\mathcal{W}_{(\kappa)}^{s}+\mathcal{W}_{(\kappa)}^{u}+$ $\mathcal{W}_{(\kappa)}^{t}=0$ explicitly, using the conditions collected so far. However, it will turn out in the next section that the general structure of the phase factors in (42), (44) and (45) provides enough constraints for deciding whether (4) can be solved.

\section{Mismatched Phases}

It turns out that already processes involving two gauge bosons and two matter fields as discussed in section 3 suffice to demonstrate the violation of WIs in TOPT.

Since all factors multiplying $c_{i} c_{j}$ and $c_{i} c_{j}^{\prime}$ in the contributions from the three channels (42), (44) and (45) are non-zero, the coefficients $c_{i}$ and $c_{i}^{\prime}$ for the $e^{+} e^{-} \gamma$ - and $3 \gamma$-vertices must be chosen such that the corresponding combination of phase factors vanishes to get $\mathcal{W}_{(\kappa)}^{s}+\mathcal{W}_{(\kappa)}^{u}+\mathcal{W}_{(\kappa)}^{t}=0$.

The overall momentum conservation $p_{1}+k_{1}=p_{2}+k_{2}$ constrains the Moyal phases. Taking these constraints into account, parameterizing the violation of energy conservation at the vertices by

$$
\delta q_{s}^{(\lambda)}=q_{s}^{(\lambda)}-p_{1}-k_{1}=q_{s}^{(\lambda)}-p_{2}-k_{2}
$$

and using an obvious notation with respect to the indices $i$ and $j$, we find in the $s$-channel

$$
\mathrm{e}^{-\mathrm{i}\left(\varphi_{i}\left(-p_{2},-k_{2}, q_{s}^{(\lambda)}\right)+\varphi_{j}\left(-q_{s}^{(\lambda)}, k_{1}, p_{1}\right)\right)}
$$




$$
=\mathrm{e}^{-\mathrm{i} p_{1} \wedge p_{2}} \mathrm{e}^{\mathrm{i} k_{1} \wedge k_{2}}\left[\begin{array}{ccc}
1 & \mathrm{e}^{-2 \mathrm{i} \delta q_{s}^{(\lambda)} \wedge p_{2}} & \mathrm{e}^{-2 \mathrm{i} \delta q_{s}^{(\lambda)} \wedge q_{s}} \\
\mathrm{e}^{2 \mathrm{i} \delta q_{s}^{(\lambda)} \wedge q_{s}} & \mathrm{e}^{2 \mathrm{i} \delta q_{s}^{(\lambda)} \wedge k_{2}} & 1 \\
\mathrm{e}^{2 \mathrm{i} \delta q_{s}^{(\lambda)} \wedge p_{1}} & \mathrm{e}^{-2 \mathrm{i} \delta q_{s}^{(\lambda)} \wedge q_{t}} & \mathrm{e}^{-2 \mathrm{i} \delta q_{s}^{(\lambda)} \wedge k_{1}}
\end{array}\right] .
$$

Analogously for the $u$-channel $\left(k_{1} \leftrightarrow-k_{2}\right)$, we obtain

$$
\mathrm{e}^{-\mathrm{i}\left(\varphi_{i}\left(-p_{2}, k_{1}, q_{u}^{(\lambda)}\right)+\varphi_{j}\left(-q_{u}^{(\lambda)},-k_{2}, p_{1}\right)\right)} \quad=\mathrm{e}^{-\mathrm{i} p_{1} \wedge p_{2}} \mathrm{e}^{-\mathrm{i} k_{1} \wedge k_{2}}\left[\begin{array}{ccc}
1 & \mathrm{e}^{-2 \mathrm{i} \delta q_{u}^{(\lambda)} \wedge p_{2}} & \mathrm{e}^{-2 \mathrm{i} \delta q_{u}^{(\lambda)} \wedge q_{u}} \\
\mathrm{e}^{2 \mathrm{i} \delta q_{u}^{(\lambda)} \wedge q_{u}} & \mathrm{e}^{-2 \mathrm{i} \delta q_{u}^{(\lambda)} \wedge k_{1}} & 1 \\
\mathrm{e}^{2 \mathrm{i} \delta q_{u}^{(\lambda)} \wedge p_{1}} & \mathrm{e}^{-2 \mathrm{i} \delta q_{u}^{(\lambda)} \wedge q_{t}} & \mathrm{e}^{2 \mathrm{i} \delta q_{u}^{(\lambda)} \wedge k_{2}}
\end{array}\right]
$$

with

$$
\delta q_{u}^{(\lambda)}=q_{u}^{(\lambda)}-p_{1}+k_{2}=q_{u}^{(\lambda)}-p_{2}+k_{1} .
$$

Finally in the $t$-channel, with

$$
\delta q_{t}^{(\lambda)}=q_{t}^{(\lambda)}-p_{1}+p_{2}=q_{t}^{(\lambda)}+k_{1}-k_{2},
$$

the phases factors are a combination of

$$
\mathrm{e}^{-\mathrm{i}\left(\varphi_{i}\left(-p_{2}, q_{t}^{(\lambda)}, p_{1}\right)+\varphi_{j}\left(k_{1},-k_{2},-q_{t}^{(\lambda)}\right)\right)}=\mathrm{e}^{-\mathrm{i} p_{1} \wedge p_{2}} \mathrm{e}^{\mathrm{i} k_{1} \wedge k_{2}}\left[\begin{array}{ccc}
\mathrm{e}^{-2 \mathrm{i} \delta q_{t}^{(\lambda)} \wedge p_{1}} & \mathrm{e}^{-2 \mathrm{i} \delta q_{t}^{(\lambda)} \wedge q_{s}} & \mathrm{e}^{-2 \mathrm{i} \delta q_{t}^{(\lambda)} \wedge p_{2}} \\
1 & \mathrm{e}^{-2 \mathrm{i} \delta q_{t}^{(\lambda)} \wedge k_{1}} & \mathrm{e}^{-2 \mathrm{i} \delta q_{t}^{(\lambda)} \wedge q_{t}} \\
\mathrm{e}^{2 \mathrm{i} \delta q_{t}^{(\lambda)} \wedge q_{t}} & \mathrm{e}^{-2 \mathrm{i} \delta q_{t}^{(\lambda)} \wedge k_{2}} & 1
\end{array}\right]
$$

and the same terms with $k_{1} \leftrightarrow k_{2}$. The phase factor $\mathrm{e}^{-\mathrm{i} p_{1} \wedge p_{2}}$ is common to all contributions. The factors $\mathrm{e}^{ \pm \mathrm{i} k_{1} \wedge k_{2}}$ must combine to a factor $\sin \left(k_{1} \wedge k_{2}\right)$ as in (47) in order to preserve the WI. This is not possible because of the remaining phase factors that depend on the TOPT momenta $\delta q_{s}^{(\lambda)}, \delta q_{u}^{(\lambda)}$ and $\delta q_{t}^{(\lambda)}$.

A simple example can serve as illustration: the dependence of the phases on the external momenta in the $s$-channel (55) and $u$-channel (56) is only the same for the factors multiplying $c_{1} c_{1}$, where the phases from TOPT have cancelled altogether by the overall energy conservation. Choosing $c_{1}=1$ and $c_{2}=$ $c_{3}=0$ would turn off all other phases in (55) and (56), but then the factors multiplying $c_{1} c_{j}^{\prime}$ in the $t$-channel (59) contain phases that depend on $\delta q_{t}^{(\lambda)}$ in a way that cannot be cancelled by other factors in the matrix elements. 
In phenomenological applications, the violation of the WI manifests itself in negative results for cross sections calculated with covariant polarization sums

$$
\sum_{\sigma= \pm} \epsilon_{\sigma}^{\mu} \epsilon_{\sigma}^{\nu *} \rightarrow-g^{\mu \nu}
$$

and including the ghost diagram

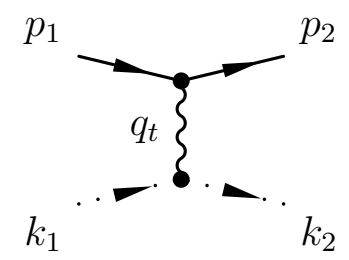

with the $\bar{c} c \gamma$ vertex given by the Faddeev-Popov Lagrangian $\mathcal{L}_{\text {g.f. }}(35)$. The pragmatic way of circumventing this problem by summing only over physical polarizations could only postpone the problem, because the negative tree level cross section will reappear as violations of the cutting rules at one-loop level.

Calculations in an effective field theory approach to NCGT [7], that consider only terms of a given finite order in $\theta_{i 0}$ are not affected by the problems discussed in this article.

\section{Conclusions}

We have shown that it is impossible to construct interaction vertices for timelike Noncommutative Gauge Theory (NCGT) in Time-Ordered Perturbation Theory (TOPT), in the form proposed in [4], that lead to scattering amplitudes with external gauge bosons which satisfy basic Ward Identities (WIs) (4). Our arguments do not depend on detailed features of the theory, but follow from phase factors that spoil the WIs. We have worked in NCQED to simplify some notations, but it is obvious that our arguments carry over to any $\mathrm{U}(N)$ time-like NCGT.

Since the definition of a physical positive norm Hilbert space for gauge theories rests on STIs and WIs, we have to conclude that TOPT cannot be used to cure the unitarity problem of time-like NCGTs. It appears that only a prescription that abrogates the relation between amputated Green functions and scattering amplitudes, as expressed by the LSZ reduction formulae, could rescue TOPT for time-like NCGT. At present, we have no suggestion for such a prescription. Since it is reasonable to insist that observed asymptotic states are described by a commuting field theory, we have doubts that such a radical modification exists. It is interesting to note in this context, that the standard reduction formulae have also been questioned for time-like NCQFT from another perspective [11]. 
An investigation of the approach of [7] in the case of time-like NCGT will be the subject of future research.

\section{Acknowledgments}

We thank Christian Schwinn and Klaus Sibold for useful discussions. This research is supported by Bundesministerium für Bildung und Forschung Germany, grants 05HT1RDA/6 and 05HT1WWA/2.

\section{References}

[1] M. R. Douglas and N. A. Nekrasov, Rev. Mod. Phys. 73 (2001) 977 arXiv:hep-th/0106048.

[2] L. Alvarez-Gaume and M. A. Vazquez-Mozo, Nucl. Phys. B 668 (2003) 293 arXiv:hep-th/0305093.

[3] J. Gomis and T. Mehen, Nucl. Phys. B 591 (2000) 265 arXiv:hep-th/0005129.

[4] Y. Liao and K. Sibold, Eur. Phys. J. C 25 (2002) 469 arXiv:hep-th/0205269.

[5] Y. Liao and K. Sibold, Eur. Phys. J. C 25 (2002) 479 arXiv:hep-th/0206011.

[6] Y. Liao and C. Dehne, Eur. Phys. J. C 29 (2003) 125 arXiv:hep-ph/0211425.

[7] J. Wess, Commun. Math. Phys. 219 (2001) 247.

[8] C. Grosse-Knetter, Phys. Rev. D 49 (1994) 6709 arXiv:hep-ph/9306321.

[9] T. Mariz, C. A. de S. Pires and R. F. Ribeiro, arXiv:hep-ph/0211416.

[10] M. Soroush, Phys. Rev. D 67 (2003) 105005 arXiv:hep-th/0302179.

[11] M. Chaichian, M. N. Mnatsakanova, A. Tureanu and Y. S. Vernov, HIP-200337-TH, arXiv:hep-th/0306158. 